\title{
Effects of Raloxifene on the Aortic Valve Function of Mice Fed with High-sugar and High-fat Diets
}

\author{
YI YANG, Z. FU1 ${ }^{1}$, CHUNYING MENG ${ }^{2}$, P. ZHAO² AND B. LEO ${ }^{2 *}$
}

Department of Gynaecology, Shenzhen People's Hospital, Jinan University, Shenzhen 518020, Guangdong, ${ }^{1}$ Department of Cardio-Thoracic Surgery, The first People's Hospital of Chenzhou, Chenzhou 423000, Hunan, ${ }^{2}$ Department of Cardiac and Vascular Surgery, Shenzhen People's Hospital, Jinan University, Shenzhen 518020, Guangdong, China

Yang et al.: Effects of Raloxifene on the Aortic Valve Function of Mice

\begin{abstract}
The current study aimed to explore the effects of raloxifene on the aortic valve function of mice fed with high-sugar and high-fat diets. C57 mice were randomly divided into five groups, 10 mice per group, normal diet group or control group, high-sugar, high-fat group, raloxifene $4.5 \mathrm{mg} / \mathrm{kg}+\mathrm{high}$-sugar, high-fat group, raloxifene $9 \mathrm{mg} / \mathrm{kg}+$ high-sugar, high-fat group, and raloxifene $18 \mathrm{mg} / \mathrm{kg}+$ high-sugar, high-fat group. Raloxifene was intragastrically administered every day for a total of three months. Steatosis and calcification of aortic valve was detected through hematoxylin and eosin staining and alizarin red staining and relative expression levels of caspase-3 and caspase-8 using immunohistochemical staining and real-time quantitative polymerase chain reaction. Compared with the high-sugar, high-fat group, both the hematoxylin and eosin staining and alizarin red staining showed that aortic valve steatosis and calcification in mice significantly improved after treatment with raloxifene, especially in the group administered with $18 \mathrm{mg} / \mathrm{kg}$. In addition, quantitative immunohistochemical staining and real-time quantitative polymerase chain reaction showed that raloxifene treatment sharply decreased mRNA expression levels of caspase-3 and caspase-8 compared to the high-sugar, high-fat group. Raloxifene at certain concentrations induced inhibitory effects on calcification and apoptosis in the aortic valves of mice after high-sugar, high-fat diet, thereby laying a foundation for therapeutic benefits raloxifene.
\end{abstract}

Key words: RAL, aortic valves, high-sugar, high-fat diet, apoptosis

With increasing aging and higher incidence of atherosclerosis, the incidence of valvular heart disease (VHD) increases annually ${ }^{[1,2]}$. Aortic valvular interstitial cells are the most abundant cells in the aortic valve of the heart and these are important in maintaining the normal structure and function of valves ${ }^{[3]}$. Valvular interstitial cells and calcium nodules are involved in the valvular degeneration and calcification. Changes in the biological functions of these cells have important effects on tissues ${ }^{[4]}$.

Raloxifene (RAL) is a second-generation selective oestrogen receptor modulator (SERM) that is initially used mainly for treating postmenopausal osteoporosis and breast cancer and later for treating cardiovascular disease. Studies have shown that, RAL and other SERMs could improve the functions of vascular endothelial cells, expand coronary arteries, regulate blood lipids, and exert protective effects on atherosclerosis, venous thrombosis, and others ${ }^{[5,6]}$. RAL regulates the functions of vascular endothelial cells through oestrogen-response elements or by other means, thereby protecting endothelial functions. RAL is expected to play a potential role in treating cardiovascular disease ${ }^{[7,8]}$.

RAL and other SERMs have been studied in basic and clinical cardiovascular diseases. These drugs were shown to improve endothelial dysfunction, increase coronary dilatation, and improve lipid profiles ${ }^{[9]}$. Aortic valve disease can be triggered by high-sugar and highfat has been confirmed ${ }^{[10]}$. It is not clear whether RAL has a dose-dependent effect on the improvement of cardiovascular disease. In the present study, a highsugar and high-fat diet mouse model was designed and different concentrations of RAL were administered for three months after high-sugar and high-fat diet with the aim of detecting the effects of RAL on aortic valve

This is an open access article distributed under the terms of the Creative Commons Attribution-NonCommercial-ShareAlike 3.0 License, which allows others to remix, tweak, and build upon the work non-commercially, as long as the author is credited and the new creations are licensed under the identical terms

Accepted 19 November 2018

Revised 18 April 2018

Received 12 April 2017

*Address for correspondence E-mail: luobin720515@126.com
Indian J Pharm Sci 2019;81(1):156-161 
steatosis, calcification, and apoptosis-related genes, laying foundation for further treating tissues with RAL.

A total of 50 male C57 mice (10 w old) were bought from Beijing Vital River. The animals were housed in a non-specific-pathogen free animal house in Shenzhen People's Hospital. The Experimental Animal Ethics Committee of Shenzhen People's Hospital approved all experimental protocols in the present study (LL-KT-201801027).

Fifty male C57BL/6 mice were randomly divided into the 5 groups of 10 mice each, normal diet group (control group), high-sugar, high-fat group, RAL $4.5 \mathrm{mg} / \mathrm{kg}+$ high-sugar, high-fat group, RAL $9 \mathrm{mg} / \mathrm{kg}+$ high-sugar, high-fat group and RAL $18 \mathrm{mg} / \mathrm{kg}+$ highsugar, high-fat group. On the day after grouping, the groups to be fed with high-sugar and high-fat diets were marked and the normal diets of these groups were replaced with high-sugar, high-fat diets. The next day, treatment group mice were intragastrically administered RAL daily for 3 mo.

Animals were sacrificed with an overdose of pentobarbital sodium after the 3 mo of RAL administration. Using RT-qPCR, the relative expression levels of caspase- 3 and caspase- 8 were detected. Aortic tissues were dissected for extracting total RNAs using a TRIzol kit (Invitrogen, USA). The concentration and purity of RNA were detected by using an ultraviolet spectrophotometer with the absorbance of $260 / 280 \mathrm{~nm}$. Reverse transcription reaction was completed using PrimeScript RT reagent kit (TaKaRa Biotechnology Co., Ltd., Shanghai, China), with every $10 \mu \mathrm{l}$ of the total system. RT-qPCR was completed using SYBR Premix Ex Taq II kit (TaKaRa Biotechnology Co., Ltd., Shanghai, China), with every $20 \mu 1$ system. PCR amplification was further completed using a quantitative PCR system (Applied Biosystems, Foster City, CA, USA). For the relative expression levels of caspase-3 and caspase-8, the standard glyceraldehyde 3-phosphate dehydrogenase (GDPDH) was used as a reference. The data was expressed by $2^{-\Delta \Delta C t}$. Differences between the samples were assessed. Primers used for caspase-3/caspase-8 and GDPDH were listed in Table 1. Aortic valve tissues were taken and fixed in $4 \%$ formaldehyde buffer for $12 \mathrm{~h}$, fully dehydrated, cleared using ethanol and xylene stepwise, and embedded in paraffin at 56-58 . Tissue slices of $5 \mu \mathrm{m}$ thickness were cut, deparaffined and placed in water for staining using hematoxylin and eosin (H and E) staining kit (Nanjing Jancheng Technology
TABLE 1: SEQUENCES OF PRIMERS FOR RTQPCR

\begin{tabular}{lc}
\hline Gene & Primer sequences \\
\hline Caspase-3 & Forward Primers 5'- \\
& TGTCATCTCGCTCTGGTACG-3' \\
Caspase- 8 & Feverse primers 5'-CCCTTTCTGCCTGTCTTCTG \\
GDPDH & $-3^{\prime}$ \\
& 5'-ACAATGCCCAGATTTCTCCCTAC-3' \\
& Reverse primers \\
& 5'-CAGACAGTATCCCCGAGGTTTG-3' \\
& Forward primers \\
& 5'-ACCTCAACTACATGGTCTAC-3' \\
Reverse primers 5'- \\
TTGTCATTGAGAGCAATGCC-3'
\end{tabular}

Co., Ltd.). Alizarin red staining was used to observe calcium deposition in the aortic tissue. For alizarin red staining, the deparaffined section was rinsed in water for $2 \mathrm{~min}$, stained with $0.1 \%$ alizarin red $\mathrm{S}$ for $30 \mathrm{~min}$, and mounted in glycerinated gelatin.

After the deparaffined section was placed in water, endogenous peroxidase was inactivated in $3 \% \mathrm{H}_{2} \mathrm{O}_{2}$ solution. Antigen retrieval was completed under high pressure or in the form of enzymatic digestion. After blocking with $5 \%$ bovine serum albumin for 30 min. Caspase-3 (GeneTex, Inc. GTX24051, USA) and caspase-8 (GeneTex, Inc. GTX60510, USA) antibodies (1:200) were added and cultured for $2 \mathrm{~h}$ at room temperature. Phosphate-buffered saline was used as a replacement for primary antibody as the negative control. A $50 \mu \mathrm{l}$ volume of ready-to-use MaxVisionTM reagent was added dropwise, and incubated at room temperature for $10 \mathrm{~min}$. 3,3'-diaminobenzidine tetrahydrochloride substrate solution was dropped into the mixture. The section was stained with hematoxylin, conventionally dehydrated, cleared, and mounted, and brown particles showed a positive signal. Image Proplus 6.0 (IPP6.0) was adopted for quantitative image analysis and determining the relative integral optical density value of different groups.

All data were processed using SPSS 17.0. The test data were represented as means \pm SD. The group treated with different concentration of RAL was compared with the control group by one-way analysis of variance, which showed statistical differences $(\mathrm{p}<0.05)$.

Caspase-3 and caspase- 8 are major apoptosis-related genes, and the expression levels of the two genes were closely related to cell apoptosis. In the present study, after 3-mo administration of RAL that, the mRNA expression levels of caspase- 3 and caspase- 8 were reduced in the aortic valves of the high-sugar, 
high-fat mice group, in particular, those in the RAL $9 \mathrm{mg} / \mathrm{kg}+$ high-sugar, high-fat group and RAL 18 $\mathrm{mg} / \mathrm{kg}+$ high-sugar, high-fat group decreased sharply (fig. 1A and B).

In observing the effects of RAL on the aortic valves of the high-sugar high-fat mice group by $\mathrm{H}$ and $\mathrm{E}$ staining, it was found that in the aortic valves of the high-sugar, high-fat mice group showed abundant dotted steatosis areas, and the aortic valve steatosis areas after the administration of different concentrations of RAL were improved to different extents, especially in the RAL18 mg/kg+high-sugar, high-fat group, in which few of such areas were found (fig. 2A-E).

Calcium deposition in aortic tissues was observed by alizarin red staining. In the aortic valve tissue of the high-sugar, high-fat mice group, numerous areas with red-dotted calcium deposition were found; however, in the group treated with different concentrations of RAL, these dotted areas are considerably diminished,

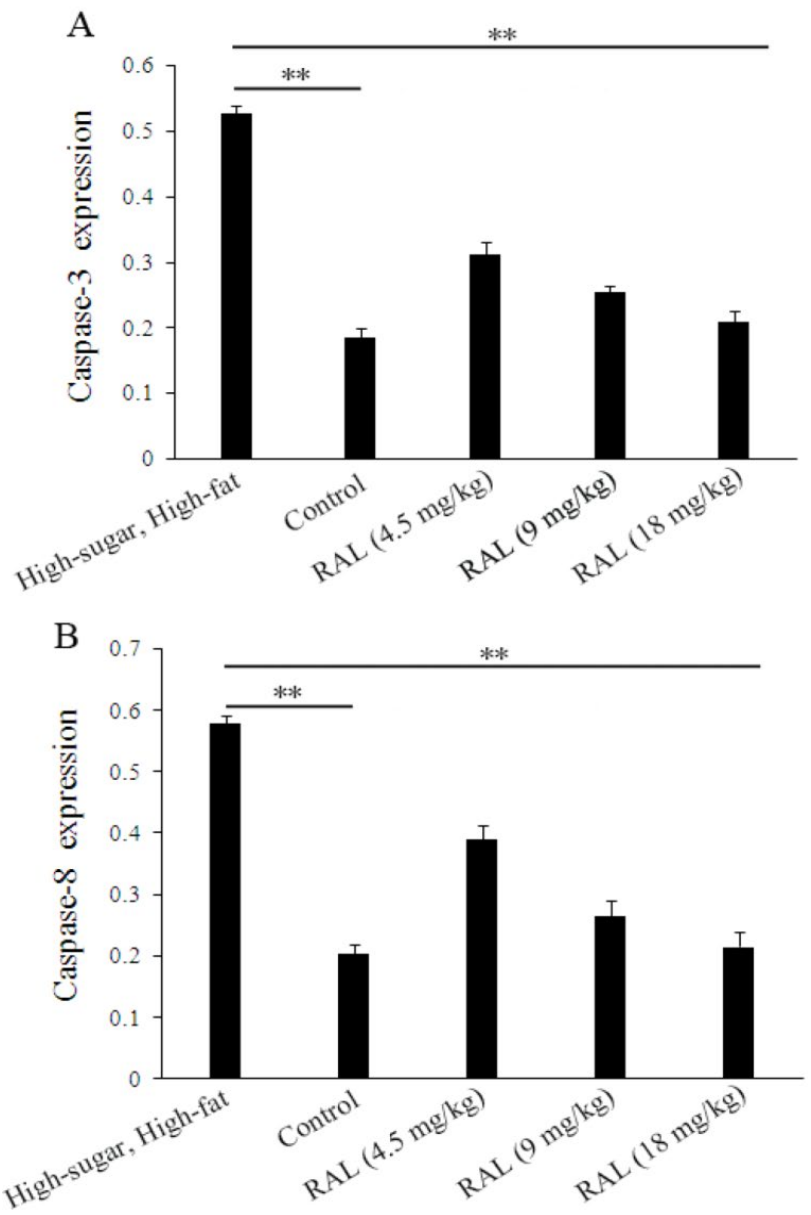

Fig.1: Effects of RAL on mRNA expression of caspase-3 and caspase-8 in the high-sugar, high-fat mice group

Effects of different concentrations of raloxifene on caspase-3 and caspase-8 mRNA expression in cardiac valves of the mice fed with high-sugar and high-fat, (A) caspase-3; (B) caspase-8, the results are expressed as mean $\pm \mathrm{SD}, * * \mathrm{p}<0.01$
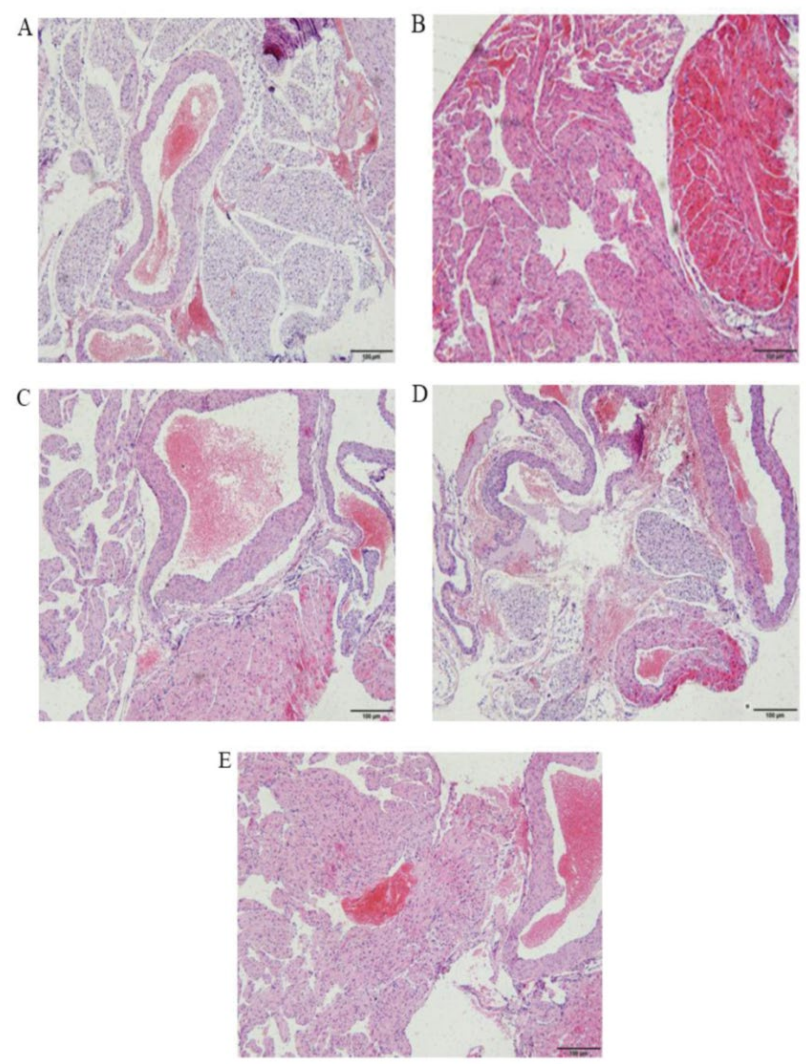

Fig. 2: Effects of RAL on the aortic valves stained with $H$ and E stain

Effects of different concentration of raloxifene on the $H$ and $E$ stained aortic valves from the high-sugar, high-fat fed mice. (A) high-sugar, high-fat group; (B) control group; (C) RAL $4.5 \mathrm{mg} /$ kg+high-sugar, high-fat group; (D) RAL $9 \mathrm{mg} / \mathrm{kg}+$ high-sugar, high-fat group; and (E) RAL $18 \mathrm{mg} / \mathrm{kg}+$ high-sugar, high-fat group

particularly in the high-concentration RAL18 $\mathrm{mg} / \mathrm{kg}$ +high-sugar, high-fat group, in which no such calcification was found (fig. 3A-E).

While analysing the expressions of caspase- 3 and caspase- 8 by immunohistochemistry, we found that the expression levels of proteins regulating the apoptosis of valvular interstitial cells after administration of RAL were inhibited to a certain extent compared with the high-sugar, high-fat group. In the sliced aortic valve tissue specimens from the high-sugar high-fat group mice, abundant positively expressed brown caspase-3 (fig. 4A-F) and caspase-8 (fig. 5A-F) were observed; in addition, through quantitative analysis of areas with positive expression, the expression levels in the highsugar, high-fat group were significantly higher than those of the group treated with RAL.

VHD is an important disease that threatens human health and advancement of age. Issues involving degenerative diseases occupy a dominant position in VHD lesions, which constitute the most common 

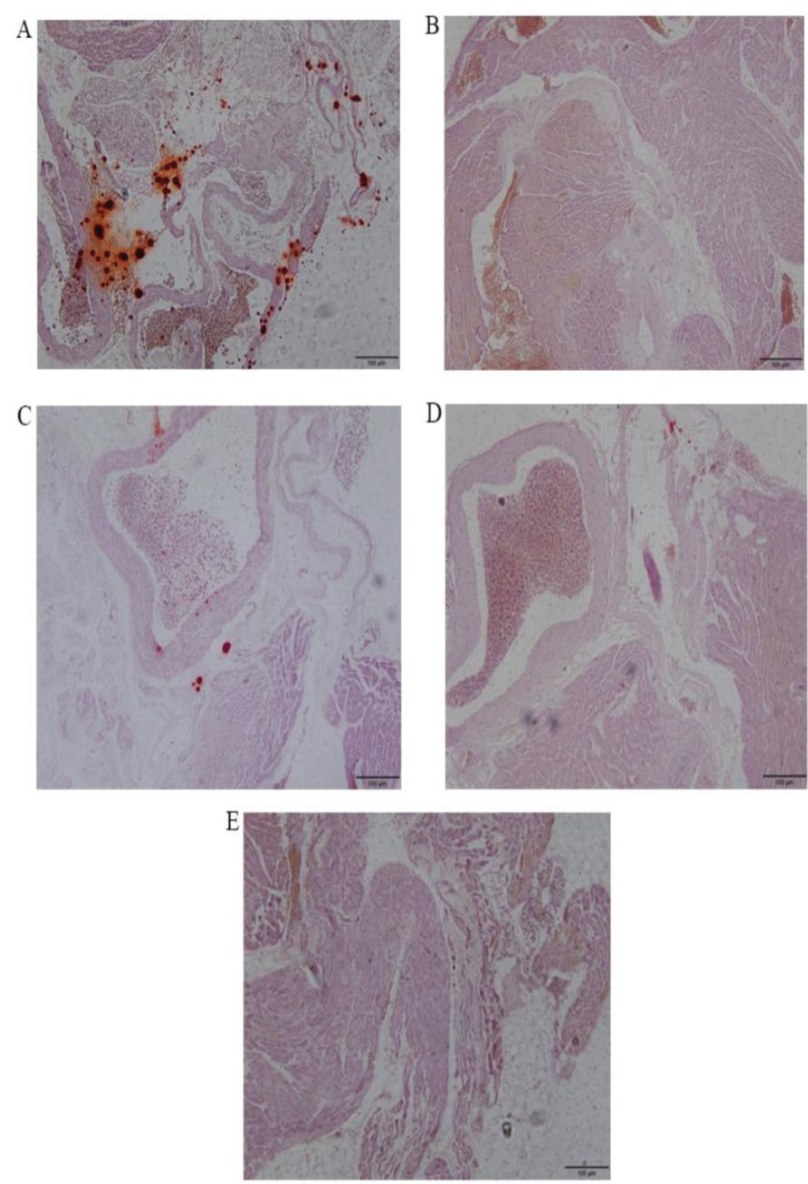

Fig. 3: Effects of RAL on the aortic valves stained with alizarin red

Effects of different concentrations of raloxifene on the alizarin red stained aortic valves from the high-sugar, high-fat fed mice group. (A) High-sugar, high-fat group; (B) control group; (C) RAL $4.5 \mathrm{mg} / \mathrm{kg}$ +high-sugar, high-fat group; (D) RAL $9 \mathrm{mg} /$ kg+high-sugar, high-fat group; and (E) RAL $18 \mathrm{mg} / \mathrm{kg}+\mathrm{high}-$ sugar, high-fat group

cause of aortic valvular disease and the most important reason for aortic valve replacement surgery ${ }^{[11,12]}$. Aortic valvular disease is a complex pathologic process. In the past years, the disease is generally considered as a passive and non-regulated process under long-term mechanical stress. At present, owing to the lack of understanding of the occurrence and development of these lesions, extremely few effective interventions are available for early valve lesions. Valvular calcification is a main pathological symptom of the disease and is an active biological process subject to active regulation ${ }^{[13]}$. In this complex regulatory process, the proliferation and apoptosis of valvular interstitial cells involve the formation of valve lesions and calcification ${ }^{[14,15]}$. Studies have shown that a high-fat diet may induce valvular lesions in New Zealand white rabbits, with significantly increased apoptosis in the lesion of the valves $^{[16]}$.
RAL belongs to the second-generation SERM group, and is mainly used initially for the treatment of post-menopausal osteoporosis and breast cancer ${ }^{[17]}$. Subsequently, the medicine was found to exert
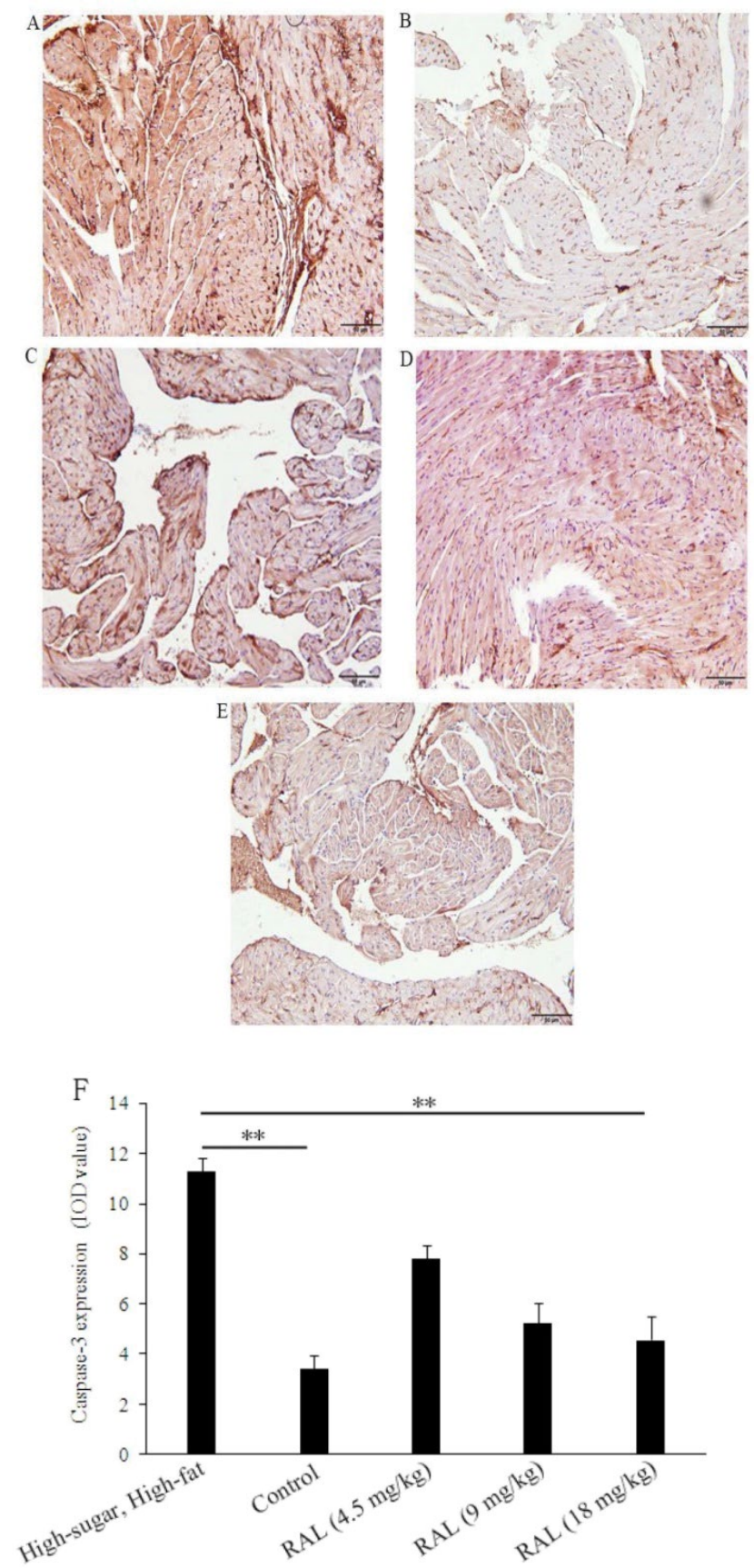

Fig. 4: Effects of RAL on caspase-3 expression levels in the aortic valves

Effects of different concentrations of raloxifene on caspase-3 expression levels in the aortic valves from the high-sugar, high-fat fed mice studied using immunohistochemistry. (A) high-sugar, high-fat group; (B) control group; (C) RAL 4.5 $\mathrm{mg} / \mathrm{kg}+$ high-sugar, high-fat group; (D) RAL $9 \mathrm{mg} / \mathrm{kg}+\mathrm{high}-$ sugar, high-fat group; and (E) RAL $18 \mathrm{mg} / \mathrm{kg}+\mathrm{high}-\mathrm{sugar}$, high-fat group and (F) results of the quantitative analysis on the caspase- 3 expression levels are expressed by means \pm SD, $* * \mathbf{p}<\mathbf{0 . 0 1}$ 

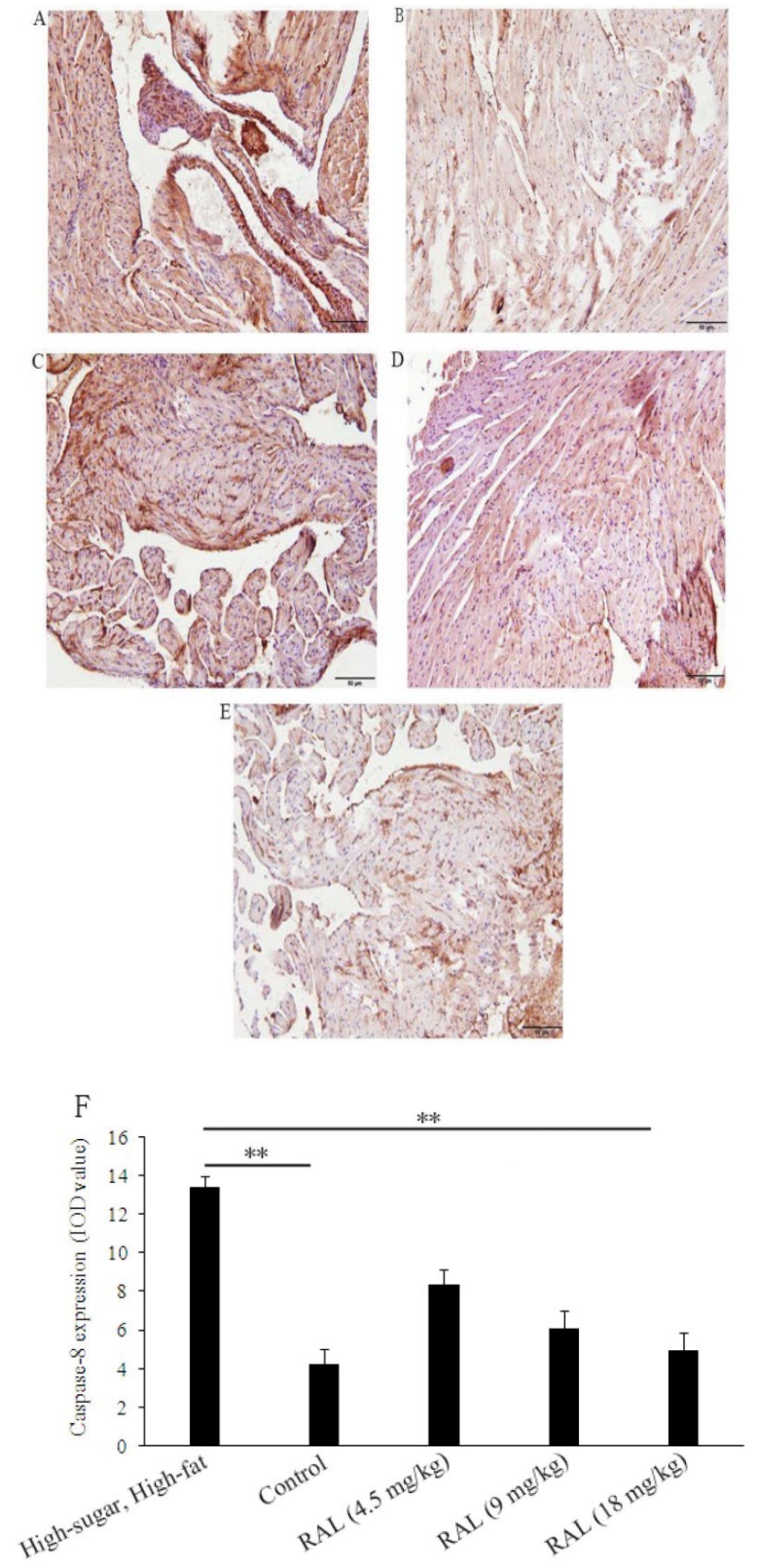

Fig. 5: Effects of RAL on the caspase-8 expression in the aortic valves

Effects of different concentrations of raloxifene on caspase-8 expression levels in the aortic valves from the high-sugar, highfat fed mice studied using immunohistochemistry. (A) Highsugar, high-fat group; (B) control group; (C) RAL $4.5 \mathrm{mg}$ / kg+high-sugar, high-fat group; (D) RAL $9 \mathrm{mg} / \mathrm{kg}+\mathrm{high}-\mathrm{sugar}$, high-fat group; and (E) RAL $18 \mathrm{mg} / \mathrm{kg}+$ high-sugar, high-fat group; (F) The results of the quantitative analysis on caspase-3 expressions are expressed as means $\pm \mathrm{SD},{ }^{* *} \mathbf{p}<0.01$

therapeutic effects on cardiovascular disease. RAL and other SERMs exhibit certain effects, such as improving the functions of vascular endothelial cells ${ }^{[18,19]}$, dilating the coronary arteries ${ }^{[20]}$, regulating the lipids, and significantly decrease soluble human vascular endothelial cadherin and increase serum total matrix metalloproteinase- 2 in healthy postmenopausal women $^{[21]}$, and protecting from atherosclerosis and venous thrombosis, and others ${ }^{[22,23]}$. RAL can improve the renal failure-induced valvular calcification by inhibiting the apoptosis of aortic valvular interstitial cells; however, the effects of RAL in animals with VHD and related clinical studies have been rarely reported ${ }^{[24]}$.

In the present study, the effects of different concentrations of RAL on the biological functions of aortic valves in the high-sugar, high-fat group of mice were discussed, and reviewed the literature and previous study summaries and designed the corresponding concentrations and length of treatment duration. After three-month administration of RAL, the mRNA expression levels in caspase- 3 and caspase- 8 in the aortic valves of the high-sugar, high-fat mice group were found to be decreased compared with those of the high-sugar, high-fat group. In particular, those of the RAL9 $\mathrm{mg} / \mathrm{kg}$ +high-sugar, high-fat group and the RAL $18 \mathrm{mg} / \mathrm{kg}$ +high-sugar, high-fat group decreased sharply. A long-term high-sugar, high-fat diet is the main cause of aortic valve steatosis. Numerous dotted steatosis areas were found in the aortic valves of the high-sugar, high-fat mice group; then, after the administration of different concentrations of RAL, the aortic valve steatosis areas were improved to varying degrees. Analysis results of the degree of calcification show numerous areas with red-dotted calcium deposition in the aortic valves of the highsugar, high-fat mice group. In comparison, these dotted areas are markedly diminished in the group treated with different concentrations of RAL; particularly, the high-concentration RAL $18 \mathrm{mg} / \mathrm{kg}$ +high-sugar, highfat group virtually showed no such calcification. As observed by quantitative analysis on regulating the protein expressions related to the apoptosis of valvular interstitial cells, the expression levels after RAL administration were also inhibited to a certain extent compared with those of the high-sugar, high-fat group.

In the present study, an aortic valve degeneration model for high-sugar, high-fat mice was established, and the effects of different concentrations of RAL on the biological functions of the aortic valves were observed. An appropriate concentration of RAL is found to exert certain inhibitory actions on tissues, thereby providing experimental reference for preventing and treating aortic valve disease with RAL.

\section{Conflicts of interest:}

The authors declare that there is no conflict of interests. 


\section{Financial support and sponsorship:}

This research was supported by the science and technology innovation fund of Shenzhen (no. JCYJ20150403101028196) and the science and technology innovation fund of Shenzhen (no.

JCYJ20140416122811926).

\section{REFERENCES}

1. Eren E, Ellidag HY, Cekin Y, Ayoglu RU, Sekercioglu AO, Yilmaz N. Heart valve disease: the role of calcidiol deficiency, elevated parathyroid hormone levels and oxidative stress in mitral and aortic valve insufficiency. Redox Rep 2014;19:34-9.

2. Talluto CJ, Silverman NH. Aortic and mitral valve stenosis with regurgitation: not due to rheumatic heart disease. Echocardiography 2011;28:E24-7.

3. Towler DA. Molecular and cellular aspects of calcific aortic valve disease. Circ Res 2013;113:198-208.

4. Carabello BA. Introduction to aortic stenosis. Circ Res 2013;113:179-85.

5. Barrett-Connor E, Mosca L, Collins P, Geiger MJ, Grady D, Kornitzer M, et al. Effects of raloxifene on cardiovascular events and breast cancer in postmenopausal women. New Engl J Med 2006;355:125-37.

6. Seli E, Pehlivan T, Selam B, Garcia-Velasco JA, Arici A. Estradiol down-regulates MCP-1 expression in human coronary artery endothelial cells. Fertil Steril 2002;77:542-7.

7. Chung MT, Cheng PY, Lam KK, Chen SY, Ting YF, Yen $\mathrm{MH}$, et al. Cardioprotective effects of long-term treatment with raloxifene, a selective oestrogen receptor modulator, on myocardial ischemia/reperfusion injury in ovariectomized rats. Menopause 2010;17:127-34.

8. O'Keefe JH, Jr., Kim SC, Hall RR, Cochran VC, Lawhorn SL, McCallister BD. Oestrogen replacement therapy after coronary angioplasty in women. J Am Coll Cardiol 1997;29:1-5.

9. Shuvy M, Abedat S, Beeri R, Valitsky M, Daher S, KottGutkowski M, et al. Raloxifene attenuates Gas6 and apoptosis in experimental aortic valve disease in renal failure. Am J Physiol Heart Circ Physiol 2011;300:H1829-H1840.

10. Drolet MC, Roussel E, Deshaies Y, Couet J, Arsenault M. A high fat/high carbohydrate diet induces aortic valve disease in C57BL/6J mice. J Am Coll Cardiol 2006;47:850-5.

11. Rajamannan NM, Evans FJ, Aikawa E, Grande-Allen KJ, Demer LL, Heistad DD, et al. Calcific aortic valve disease: not simply a degenerative process: A review and agenda for research from the National Heart and Lung and Blood
Institute Aortic Stenosis Working Group. Executive summary: Calcific aortic valve disease-2011 update. Circulation 2011;124:1783-91.

12. Zigelman CZ, Edelstein PM. Aortic valve stenosis. Anesthesiol Clin 2009;27:519-32.

13. Mulholland DL, Gotlieb AI. Cell biology of valvular interstitial cells. Can J Cardiol 1996;12:231-36.

14. Cowell SJ, Newby DE, Prescott RJ, Bloomfield P, Reid J, Northridge DB, et al. A randomized trial of intensive lipidlowering therapy in calcific aortic stenosis. N Engl J Med 2005;352:2389-97.

15. Rossebo AB, Pedersen TR, Boman K, Brudi P, Chambers JB, Egstrup $\mathrm{K}$, et al. Intensive lipid lowering with simvastatin and ezetimibe in aortic stenosis. N Engl J Med 2008;359:1343-56.

16. Kumar A, Wiltz DC, Grande-Allen KJ. Gentamicin Reduces Calcific Nodule Formation by Aortic Valve Interstitial Cells In Vitro. Cardiovasc Eng Technol 2013;4:16-25.

17. Davies GC, Huster WJ, Lu Y, Plouffe L, Jr., Lakshmanan M. Adverse events reported by postmenopausal women in controlled trials with raloxifene. Obstet Gynecol 1999;93:558-65.

18. Lamas AZ, Caliman IF, Dalpiaz PL, de Melo AF, Jr., Abreu GR, Lemos EM, et al. Comparative effects of estrogen, raloxifene and tamoxifen on endothelial dysfunction, inflammatory markers and oxidative stress in ovariectomized rats. Life Sci 2015;124:101-9.

19. Sumino H, Ichikawa S, Kasama S, Takahashi T, Sakamoto $\mathrm{H}$, Koizumi A, et al. Effects of raloxifene on brachial arterial endothelial function, carotid wall thickness, and arterial stiffness in osteoporotic postmenopausal women. Int Heart J 2010;51:60-7.

20. Meyer MR, Prossnitz ER, Barton M. The G proteincoupled estrogen receptor GPER/GPR30 as a regulator of cardiovascular function. Vascul Pharmacol 2011;55:17-25.

21. Christodoulakos GE, Lambrinoudaki IV, Botsis DC. The cardiovascular effects of selective estrogen receptor modulators. Ann N Y Acad Sci 2006;1092:374-84.

22. Gradishar W, Glusman J, Lu Y, Vogel C, Cohen FJ, Sledge GW Jr. Effects of high dose raloxifene in selected patients with advanced breast carcinoma. Cancer 2000;88:2047-53.

23. Poirot M, Silvente-Poirot S, Weichselbaum RR. Cholesterol metabolism and resistance to tamoxifen. Curr Opin Pharmacol 2012;12:683-9.

24. Wiernicki T, Glasebrook A, Phillips DL, Singh JP. Estrogen and a novel tissue selective estrogen receptor modulator raloxifene directly modulate vascular smooth muscle cell functions: Implications in the cardioprotective mechanism of estrogen. Circulation 1996;94:1615-5. 